\title{
Manasement of a mixed treatment technique in a patient with a class I bimaxillary protrusion
}

\author{
B. Delvallez, O. Sorel
}

\section{INTRODUCTION}

Orthodontic treatment using a mixed technique (maxillary lingual appliance and mandibular buccal appliance) increases the difficulties of detailing the occlusal finishing. We will see the implementation of the various methods used to treat the following Class I skeletal case, with dento-maxillary discrepancy and a previously treated periodontal disease.

\section{ETIOLOGY AND DIAGNOSIS}

The patient came for a consultation to the Rennes DFO Center in January 2008 for the purpose of aligning her teeth and lessening her concern about her "teeth being too far forward."

The diagnosis was:

Functional: a slight lingual thrust in the maxilla during swallowing. There is no problem with breathing and she was tested negative for OSA.

Extra-oral: in the facial view note the rightleft asymmetry with the right side less developed. The smile is broad and asymmetrical. In the profile view the naso-labial angle is closed with the presence of a bimaxillary protrusion (Figs. 1 and 2).

Skeletal: in the transverse direction the A-P cephalometric xray does not indicate any abnormality, in the sagittal direction, the cephalometric analysis shows a skeletal Class I and a bimaxillary protrusion (1/NA $37^{\circ}$ and i/NB $34^{\circ}$ and an IMPA of $100^{\circ}$ ) (Fig. 3).

Dento-alveolar: the patient is an Angle Class | with a $2 \mathrm{~mm}$ Class || right canine. 


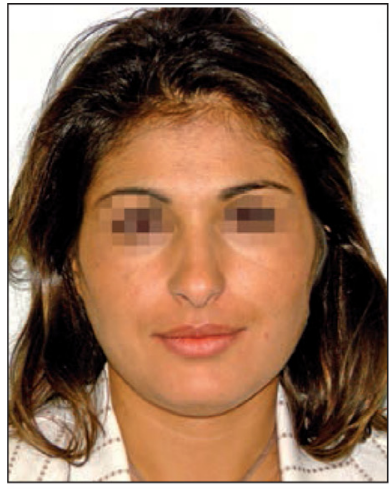

a

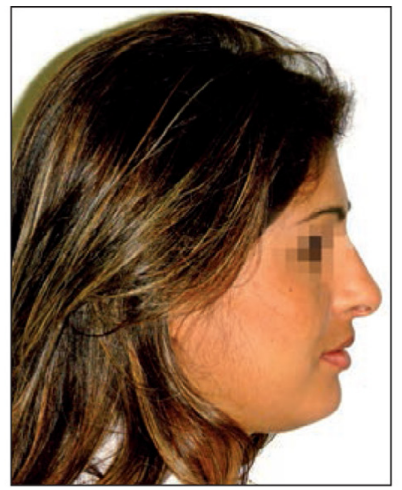

b

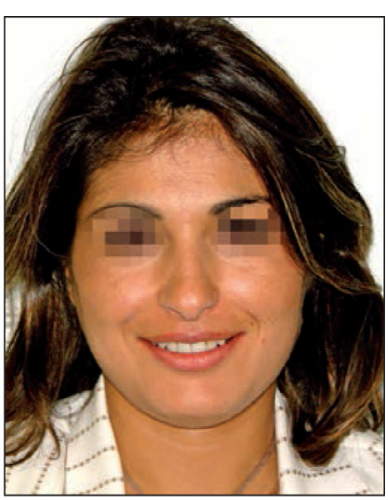

C

Figure 1

Facial photographs before treatment: frontal, profile and smile.
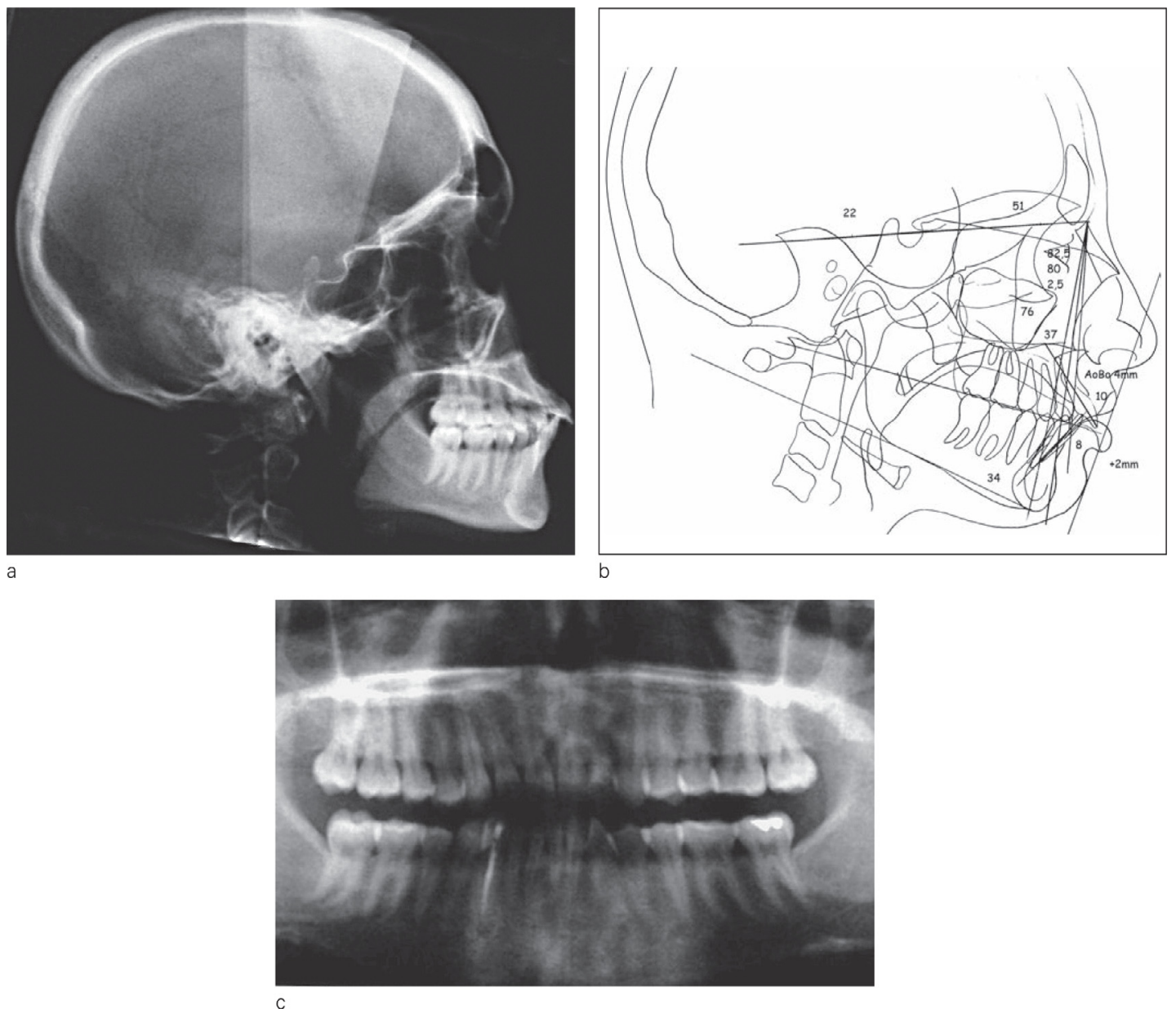

Figure 2

(a) Profile cephalometric xray (b) Steiner Analysis and (c) pre-treatment orthopantomogram. 

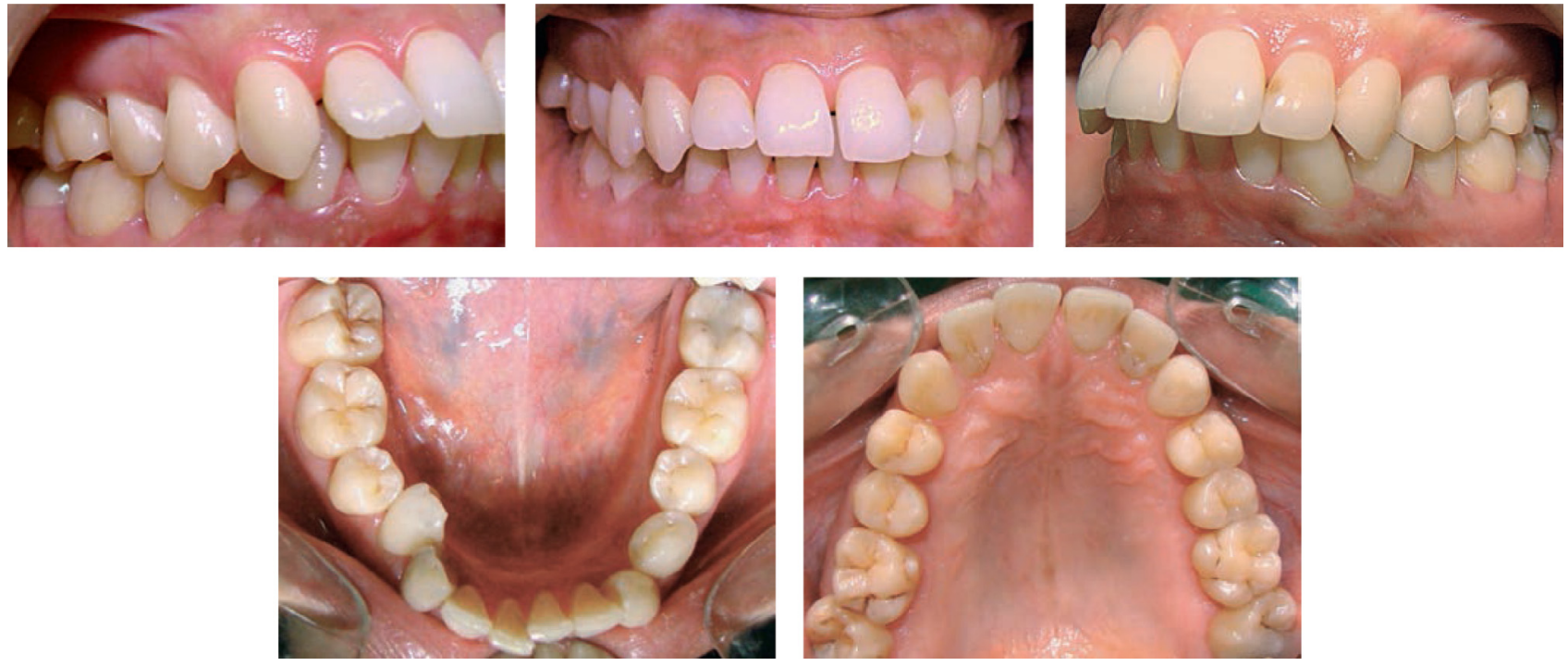

Figure 3

Intra-oral photographs before treatment.
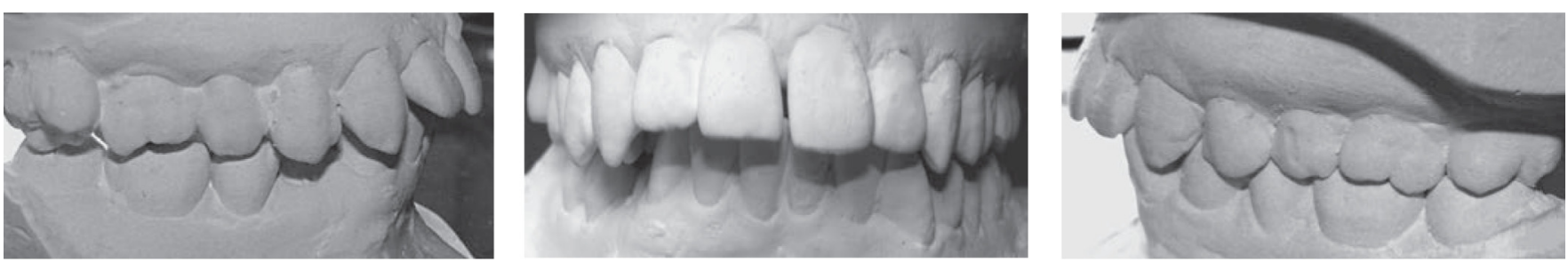

Figure 4

Study models before treatment.

The incisal overjet is $6 \mathrm{~mm}$ and the overbite is $5 \mathrm{~mm}$.

There exists two local crossbites between 17 and 47 in one case (17 is buccal) and in the other case between 14 and 44 in the other case (44 is in lingually tipped) (Figs. 3 and 4). There is $6 \mathrm{~mm}$ lower dental crowding with abnormal crown morphology on 34 and 44 . In addition, the exam shows a moderate vertical alveolar bone loss (defect) due to a previously treated periodontal disease.

\section{TREATMENT OBJECTIVES}

It was decided to perform treatment with the extraction of four first premolars. This decision is justified by: the maxillo-mandibular protrusion and the desire of the patient to not have protrusive teeth as well as the dental 
crowding and the abnormal shapes of 34 and 44, and finally by a weak periodontium even though the periodontal pathology had been treated and stabilized.

The extractions of the premolars will enable us to create the necessary space for alignment with a favorable lingual and palatal tipping of the maxillary and mandibular incisors. In order to avoid reducing the tongue space and to not modify the profile,

\section{TREATMENT PROGRESSION}

Treatment started in June 2008. The extractions of the 4 premolars were done after placement of the maxillary lingual system in June 2008 (Fig. 5).

The leveling and aligning phase was completed in 7 months. The beginning of mesialization of the mandibular second premolars (by sliding mechanics using elastomeric chains) was accompanied by mesio-lingual rotations. This was due to a mechanical error (missing metal ligature on

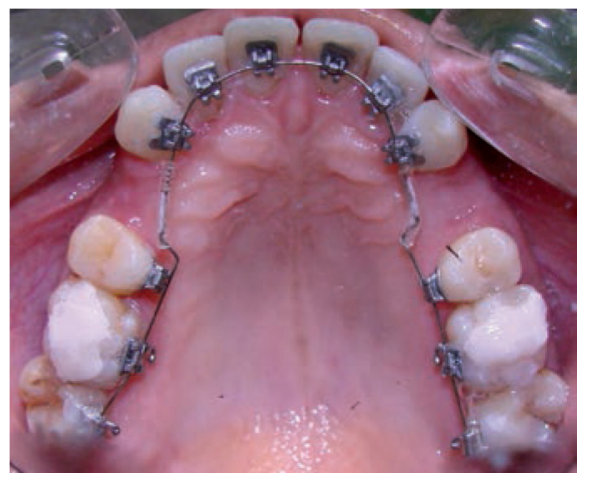

Figure 5

Photograph of the maxillary arch after bonding. we did not use a special anchorage system.

The right and left excursions are disturbed by the completely blocked out 17 that leads to a significant interference and prematurity in opening and closing. Repositioning of this tooth made it possible to improve occlusal function of mastication.

It was necessary to retrain swallowing in parallel with exercises as described by Garliner or Fournier.

the 5 elastomeric chains holding the 4 tie wings of the bracket, under-sized wire and of course a force that did not pass through the center of resistance leading to the rotation).

During the first re-evaluation at the end of 1 year, we note: a mild retraction of the maxillary incisors without noticeable change in their inclination, and a lingual tipping of the mandibular incisors (according to superimposition using the the LVD method as confirmed by the Steiner Analysis) (Fig. 6).

Fortunately, we reacted quickly and changed mechanics. Orthodontists have to be very careful with mechanics because the unwanted rotation took place in one month! The space closure was done with an .020 Australian wire that was rigid enough with low friction with no control over the inclination of the mandibular incisors, the object of which was to obtain a linguoversion of the incisors (Fig. 7).

We then began maxillary incisor retraction and had some difficulties in 


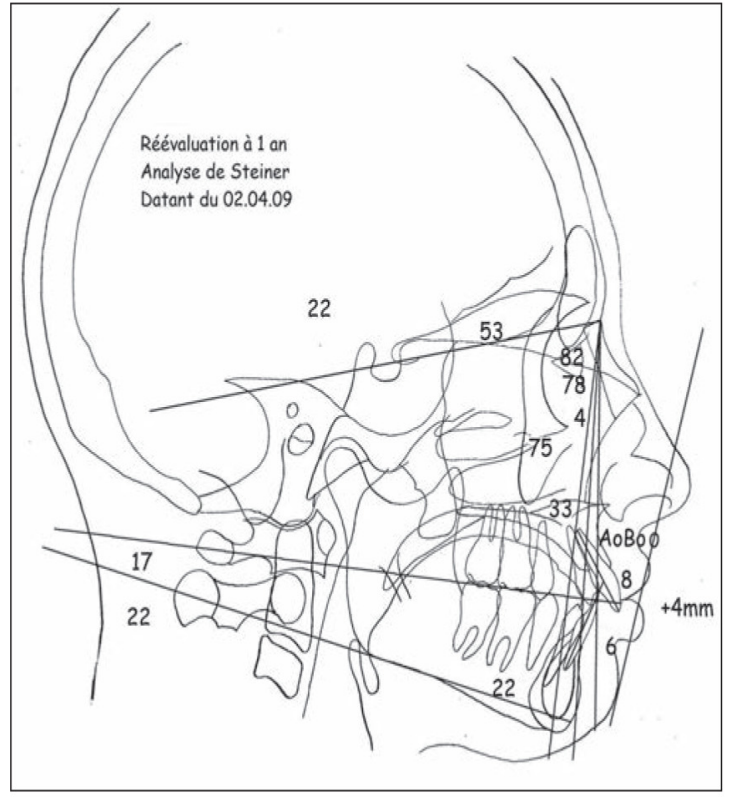

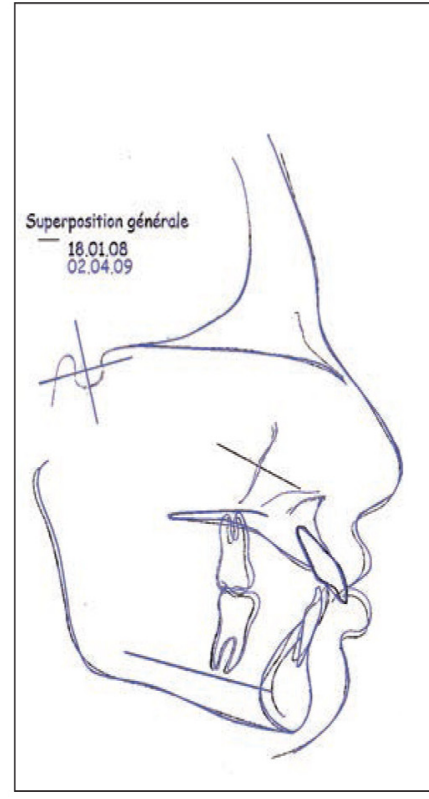

b

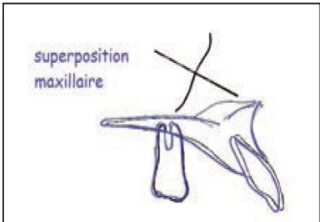

C

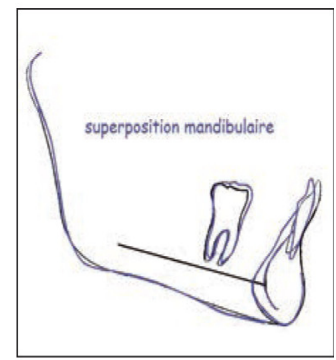

d

Figure 6

(a) Steiner Analysis and (b) Superimpositions at 1 year of treatment.
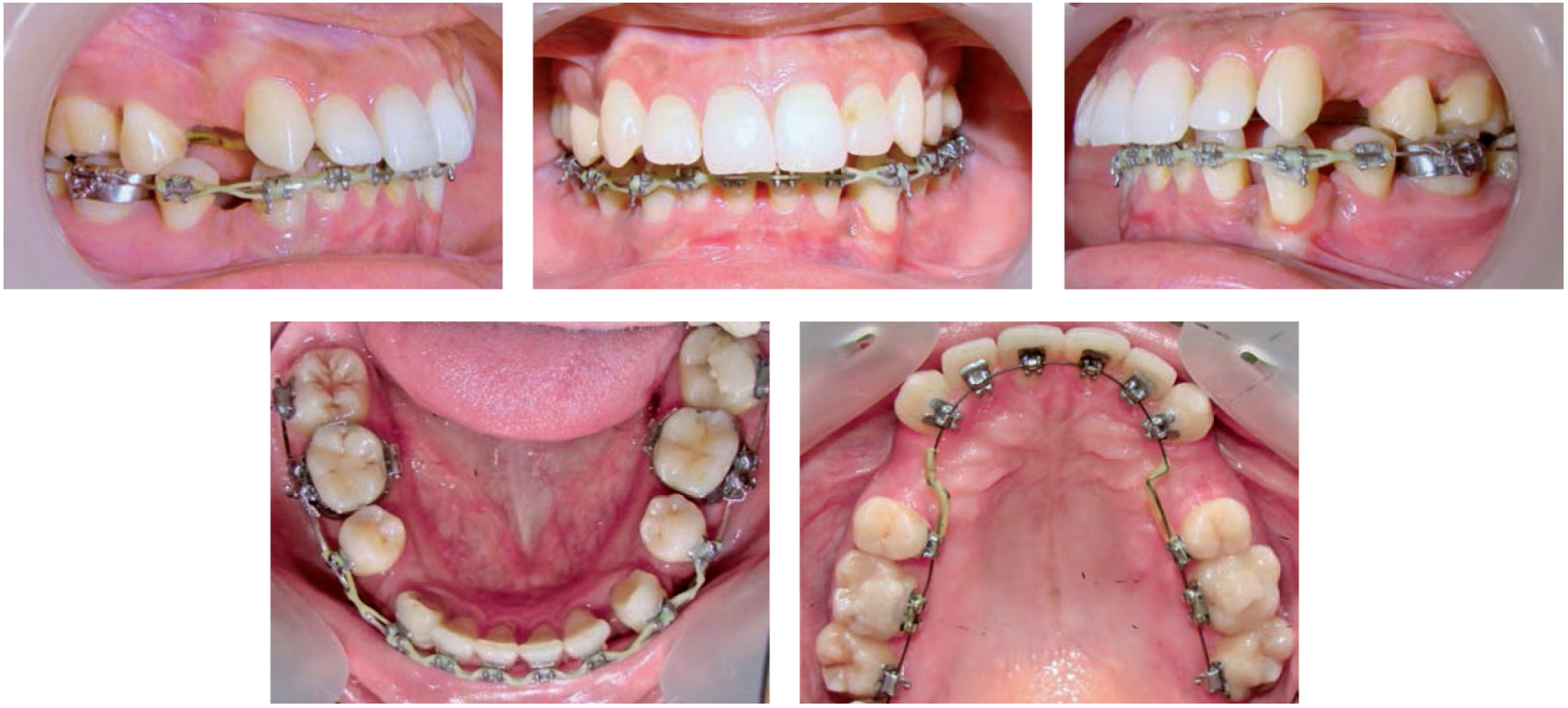

Figure 7

Intra-oral photographs during the closure of the mandibular spaces. 

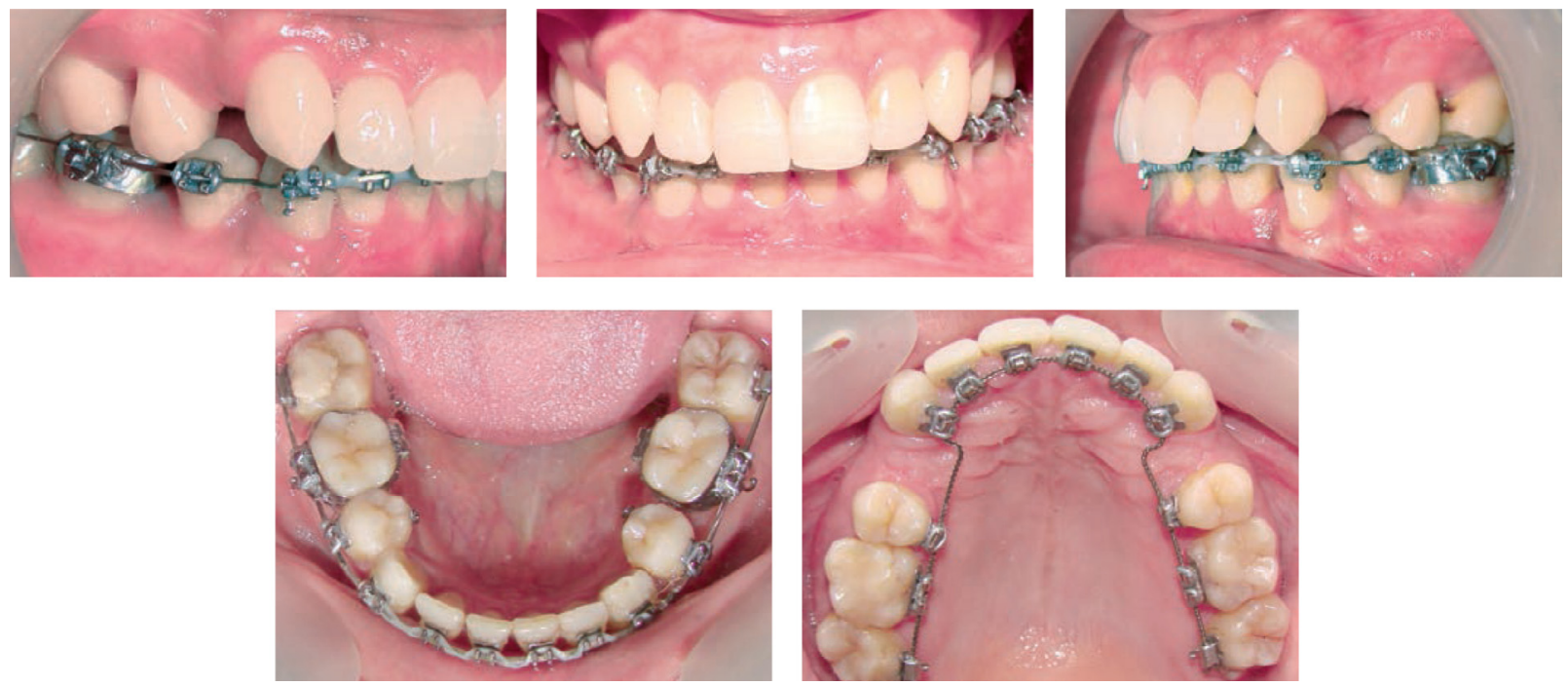

Figure 8

Intra-oral photographs at the beginning of the closure of the maxillary extraction spaces.
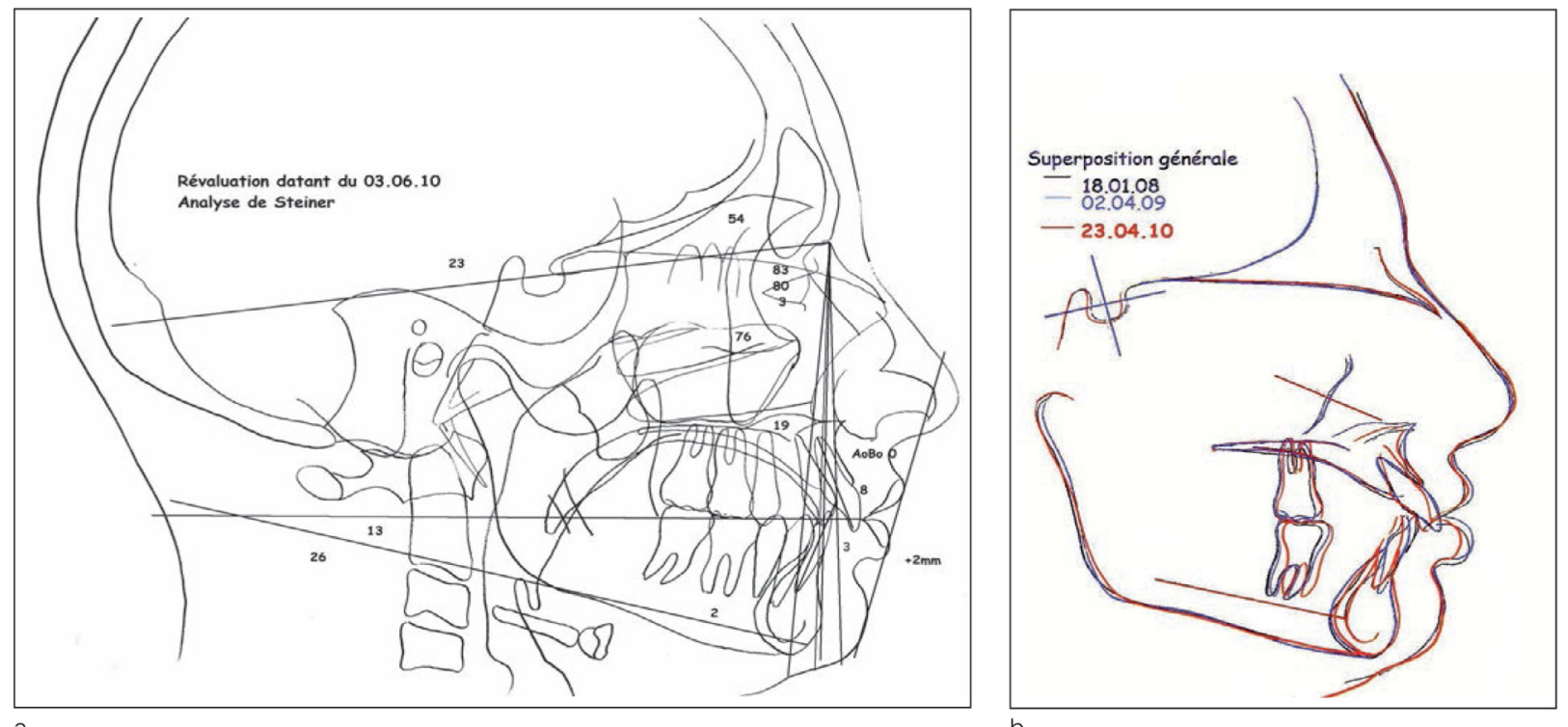

Figure 9

(a) Steiner Analysis at 2 years of treatment and (b) superimposition at 2 years of treatment. 

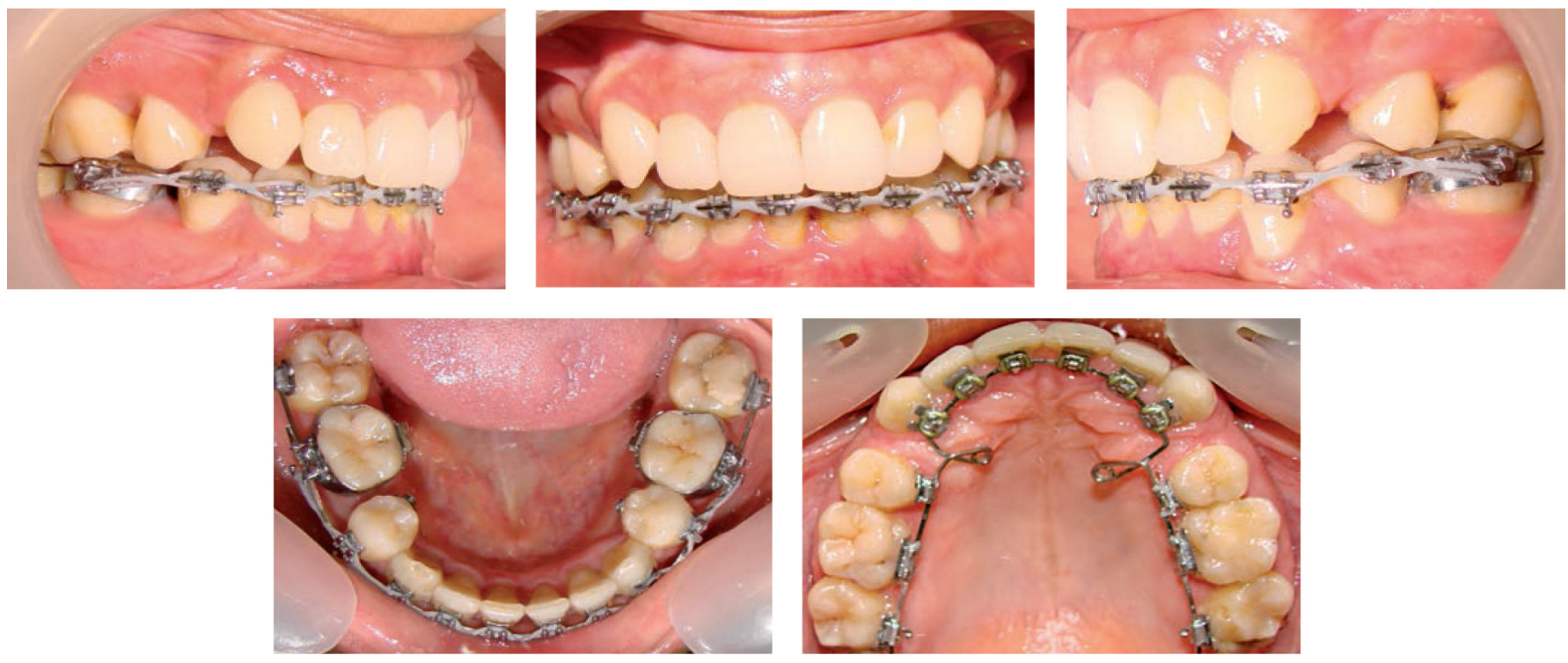

Figure 10

Intra-oral photographs during closure of the maxillary extraction spaces.

controlling the inclination of the maxillary incisors (Figs. 8 and 9).

Then after accelerating the maxillary space closure and having overcome the friction, we incorporated retraction loops. (Fig. 10).

Class II traction elastics (thin diameter) were worn at night between the mandibular first molars and the maxillary second premolars in order to limit the mesialization of the maxillary first molars (Fig. 11).

Thereafter, the end of the maxillary space closure was achieved by using mini screw anchorage and by removing the mandibular system. Since the patient can no longer wear intermaxillary elastics, we placed the screws to accomplish single jaw retraction. Buccal brackets became troublesome for the retraction of the maxillary incisor-canine group. (Fig.12).
Criss-cross elastics were placed to reduce the crossbite of 15-25. A coronoplasty was performed on 21 (on the occlusal edge) in order to make the shape of 21 and 11 symmetrical, also on 13 and 23 to reduce the the cuspid tip, that appeared too pronounced for a woman. The debanding took place at the end of 2011.

The superimpositions at the end of treatment, in violet, show a little too much retroclination of the maxillary incisors. The lingual tipping and the retraction of the mandibular incisors and the mesialization of the maxillary first molars. The upper and lower lips, also, retracted (Figs 13-15). We bonded a nickel-titanium braided wire in the maxilla and mandible and had her wear a Hawley plate with the goal of avoiding any reopening of any extraction spaces in the maxilla. 

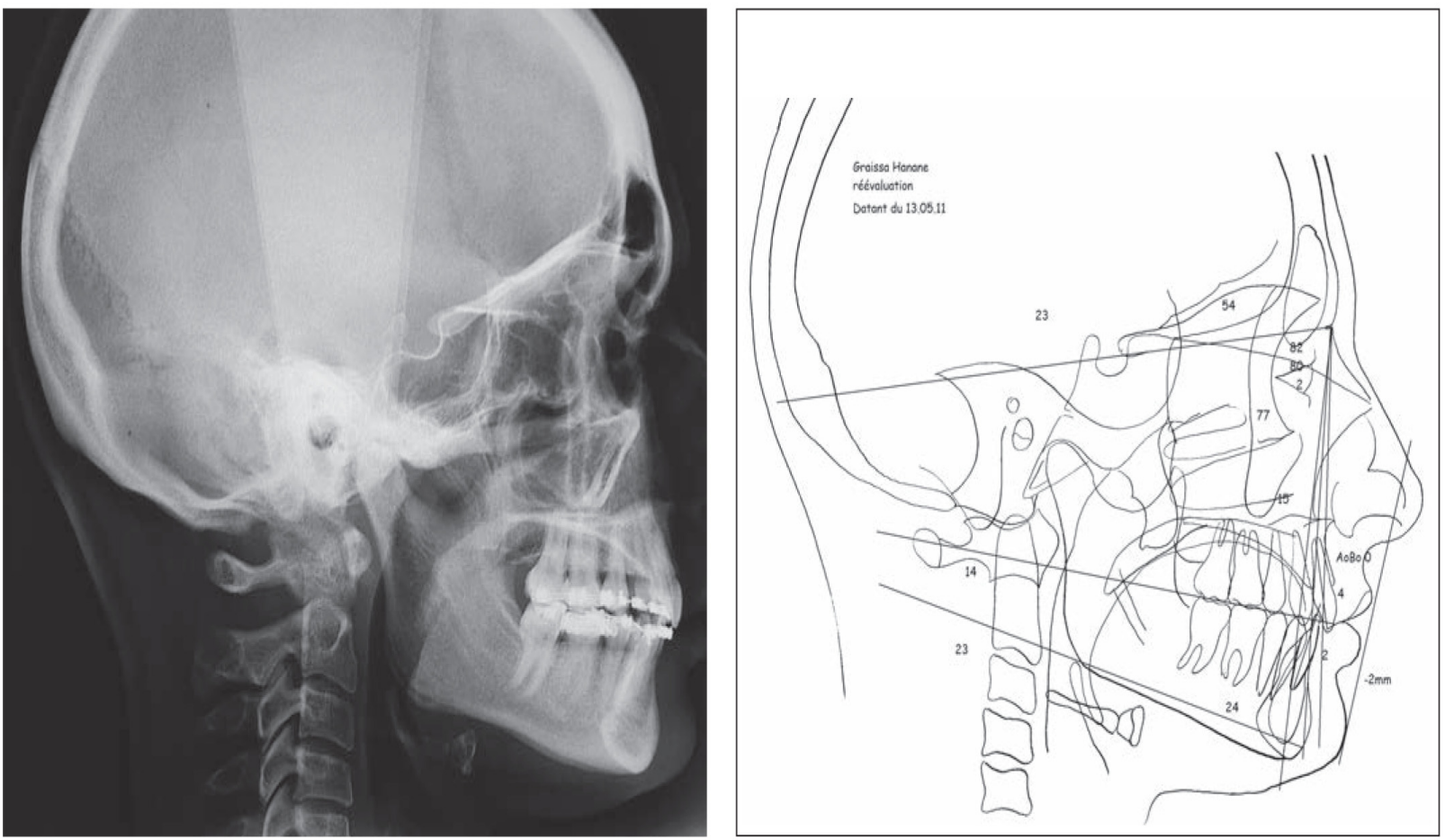

a

b

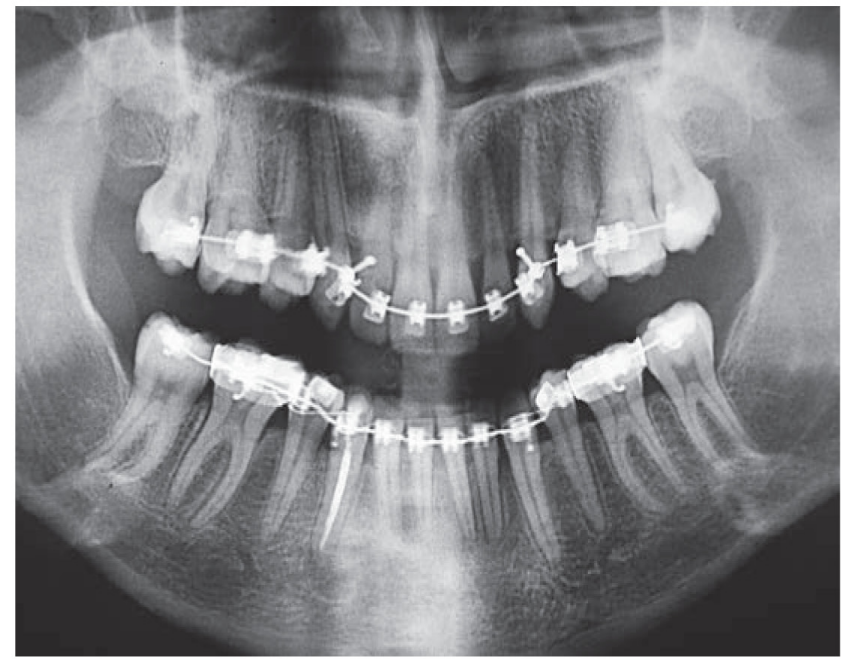

c

Figure 11

Radiographs prior to placement of the mini screws at three years of treatment. 

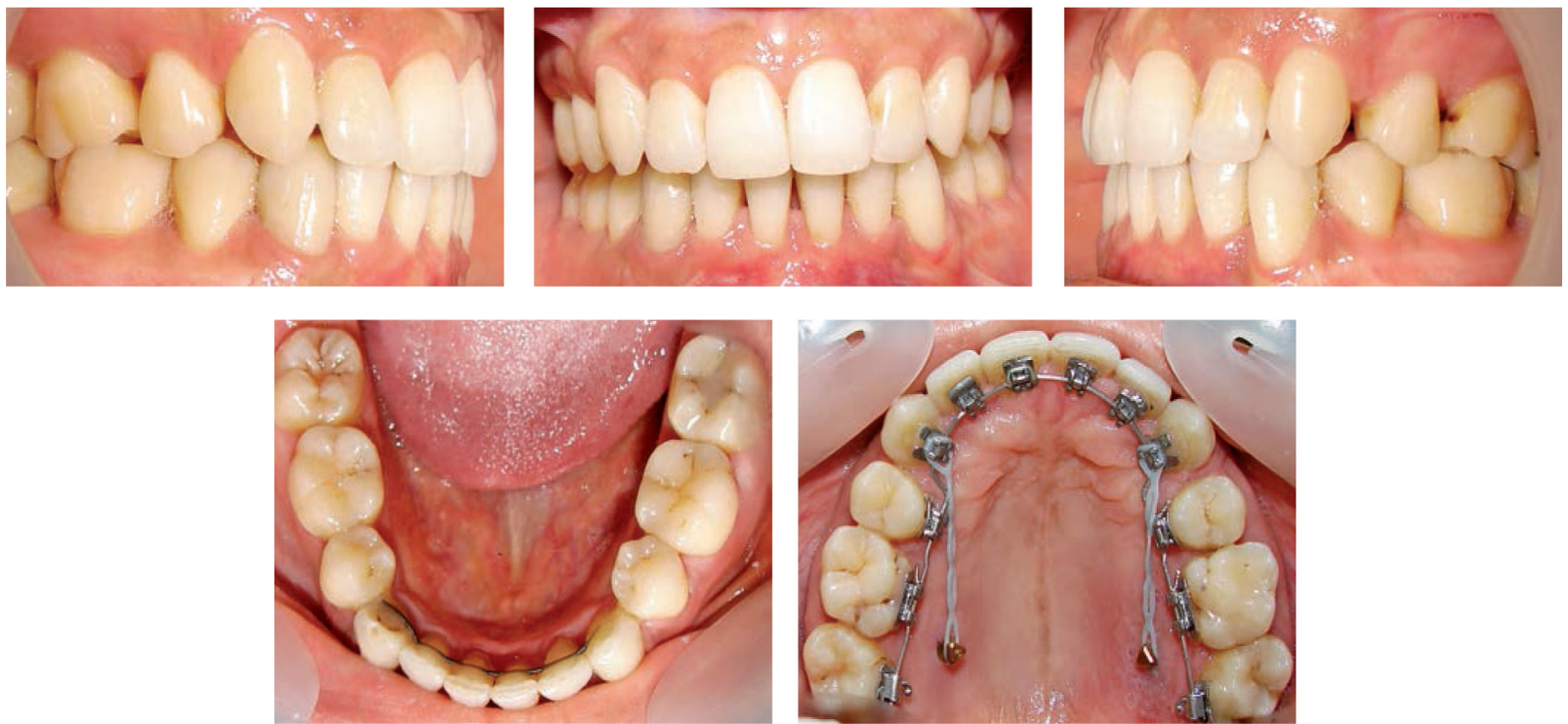

Figure 12

Intra-oral photographs at the end of maxillary space closure with mini-screws.
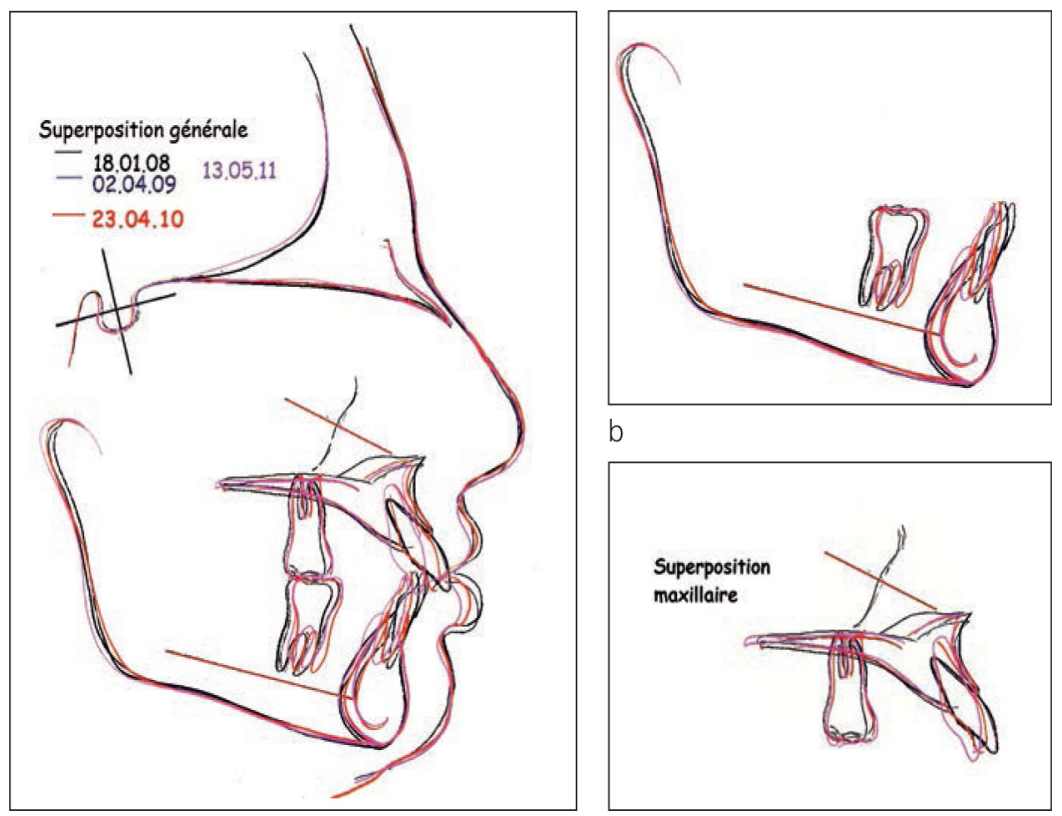

$b$

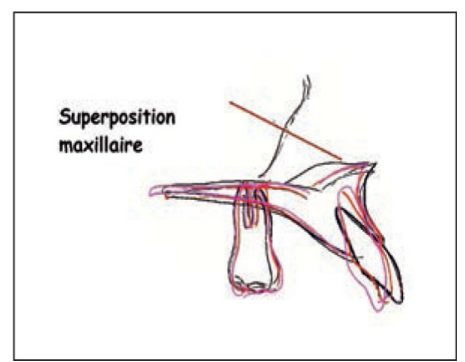

C

Figure 13

Superimposition at the end of treatment. 

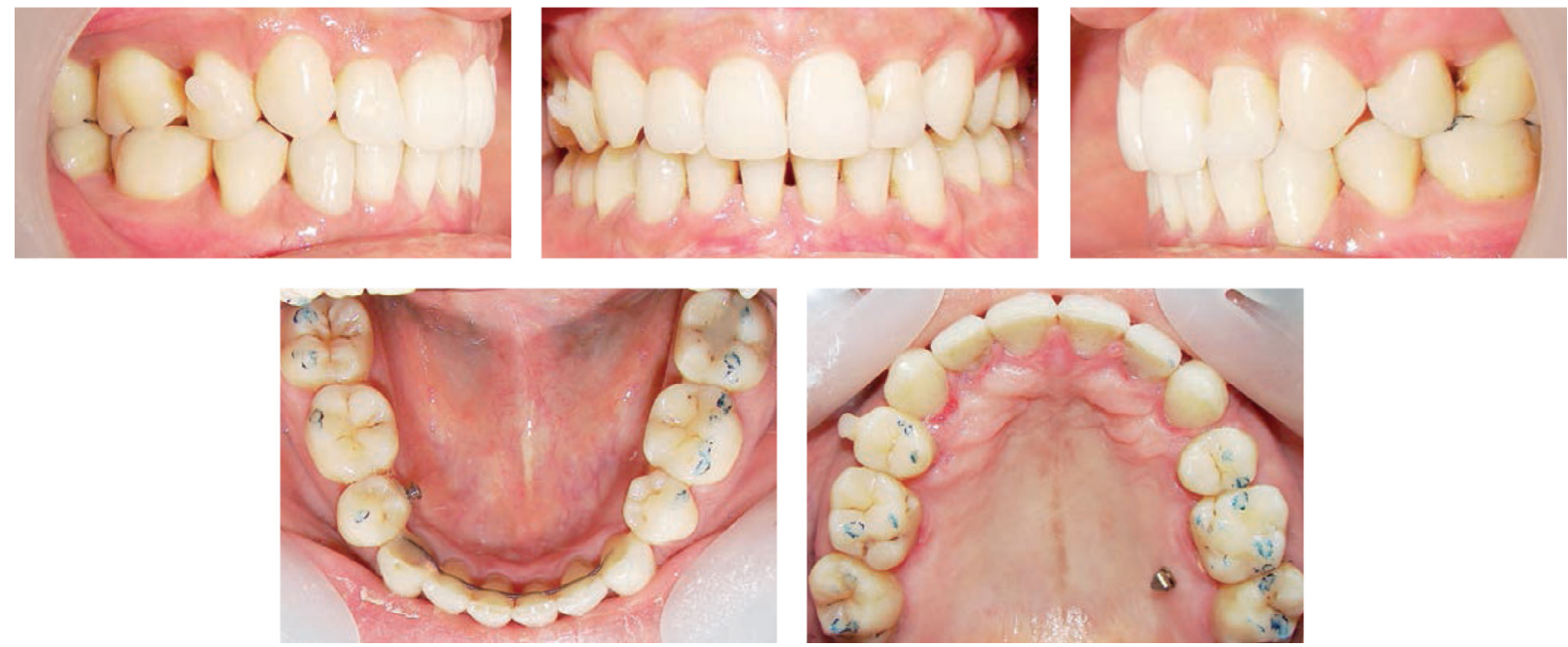

Figure 14

Intra-oral photographs at the end of treatment.
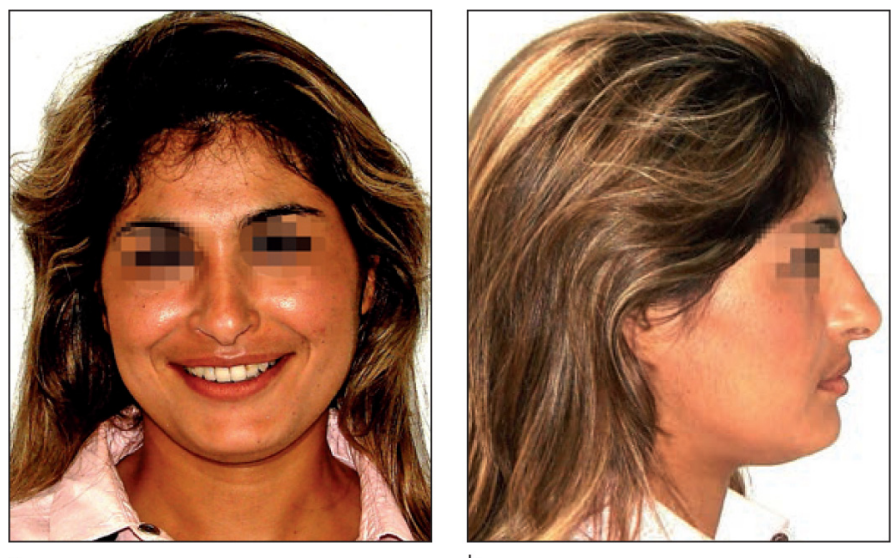

b

Figure 15

Profile and smile photographs.
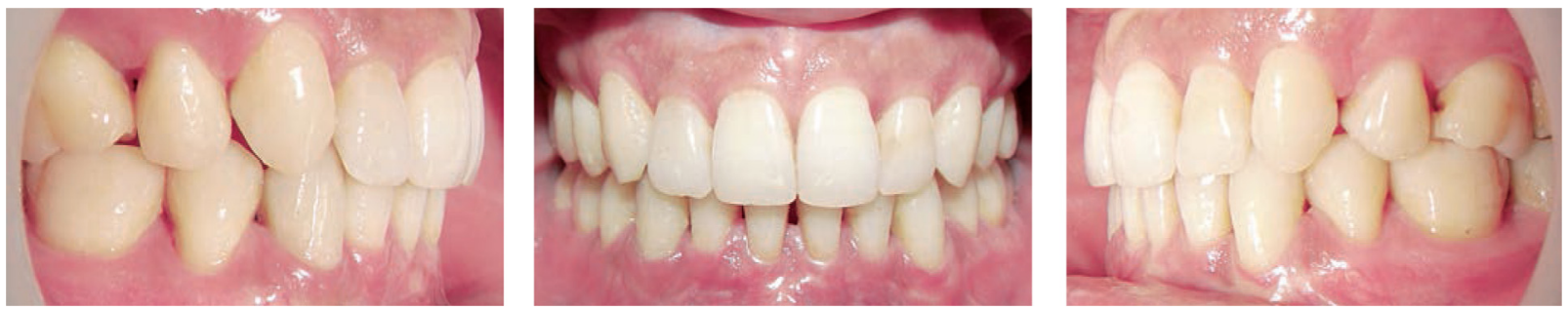

Figure 16

Intra-oral photographs and the end of retention. 

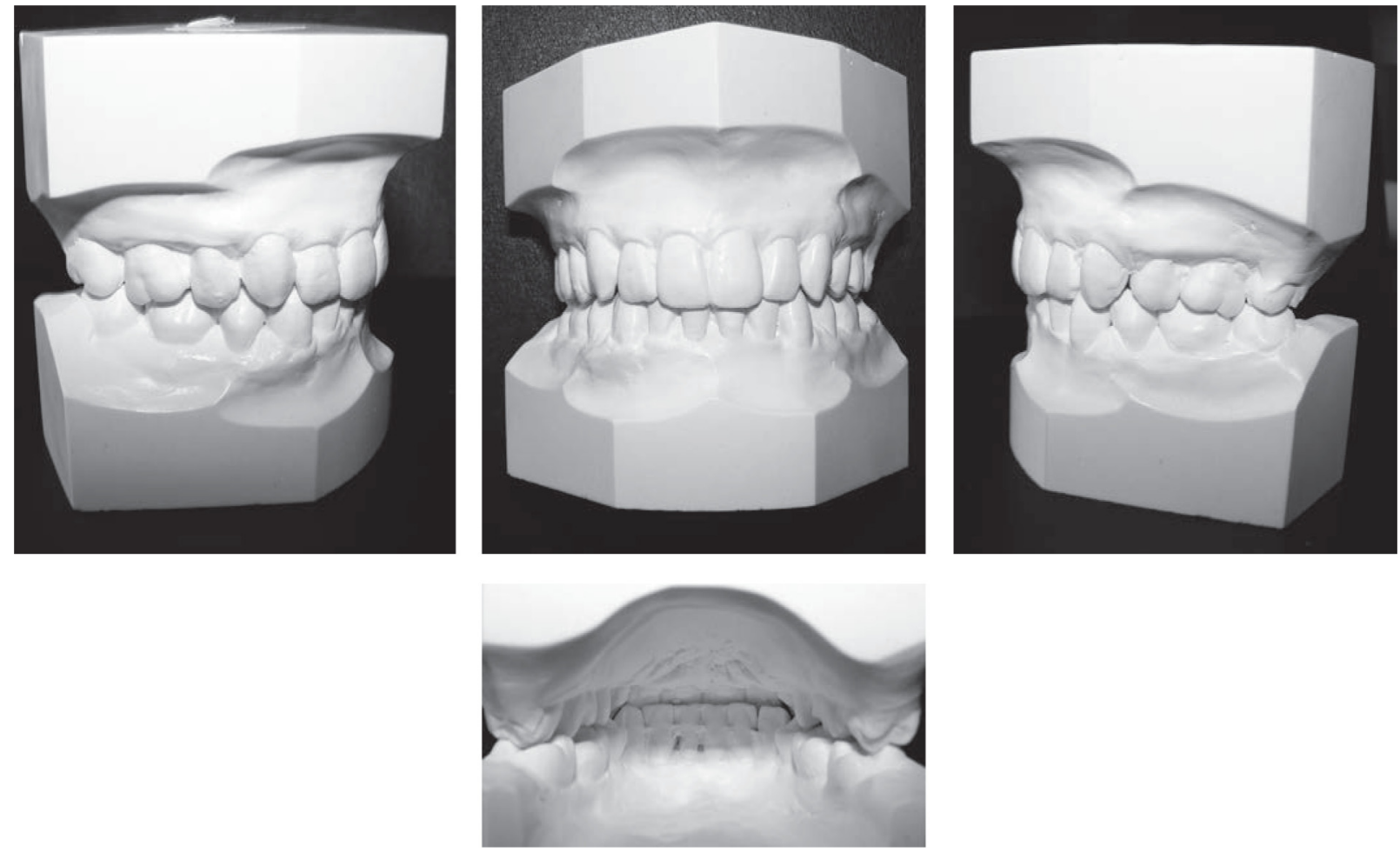

Figure 17

Models at the end of retention. 